Case Report

\title{
Rothia mucilaginosa Meningitis in a Child with Myelodysplastic Syndromes
}

\author{
Fumihiro Ochi (D), ${ }^{1}$ Ryota Nakamura, ${ }^{1}$ Reiji Miyawaki, ${ }^{1}$ Kyoko Moritani, ${ }^{1}$ \\ Shinobu Murakami, ${ }^{2}$ and Hisamichi Tauchi ${ }^{1}$ \\ ${ }^{1}$ Department of Pediatrics, Ehime University Graduate School of Medicine, Toon, Ehime, Japan \\ ${ }^{2}$ Clinical Laboratory Division, Ehime University Hospital, Toon, Ehime, Japan \\ Correspondence should be addressed to Fumihiro Ochi; fmochi2000@yahoo.co.jp
}

Received 15 March 2021; Revised 22 August 2021; Accepted 7 September 2021; Published 13 September 2021

Academic Editor: John B. Amodio

Copyright ( 92021 Fumihiro Ochi et al. This is an open access article distributed under the Creative Commons Attribution License, which permits unrestricted use, distribution, and reproduction in any medium, provided the original work is properly cited.

\begin{abstract}
Rothia mucilaginosa is a Gram-positive coccus and an opportunistic pathogen in immunocompromised hosts. The microorganism has been implicated in serious infections, including bacteremia meningitis or endocarditis. However, there is a dearth of investigations on meningitis, especially in children. As this infection is rare and only a few cases have been recorded, evidencebased guidelines for adequate infection treatment are lacking. We herein report the case of a 12-year-old boy with myelodysplastic syndromes (MDS) presenting with a change in mental status who was diagnosed as having febrile neutropenia and bacterial meningitis caused by $R$. mucilaginosa at 23 days after unrelated cord blood transplant. In our case, the minimum inhibitory concentrations (MICs) of meropenem and vancomycin (VCM) were both $\leq 1 \mu \mathrm{g} / \mathrm{mL}$, whereas the MIC of daptomycin (DAP) was $4 \mu \mathrm{g} / \mathrm{mL}$. The patient was treated with intravenous antimicrobial therapy due to meropenem for 43 days because he had febrile neutropenia. During follow-up, the patient had no neurological complications. We retrospectively reviewed the antimicrobial susceptibility of all R. mucilaginosa isolates $(n=5)$ from blood or cerebrospinal fluid cultures at our hospital. The MIC of VCM was $<0.5 \mu \mathrm{g} / \mathrm{mL}$ for all strains, whereas the MIC of DAP was $\geq 2 \mu \mathrm{g} / \mathrm{mL}$ for all strains. The MIC of MEPM was $>1 \mu \mathrm{g} / \mathrm{mL}$ for one strain. We recommend choosing VCM as the primary treatment for invasive $R$. mucilaginosa infections until antimicrobial susceptibility results are known, especially in immunocompromised children.
\end{abstract}

\section{Introduction}

Rothia mucilaginosa, previously known as Stomatococcus mucilaginosus, is a Gram-positive and coagulase-negative coccus occurring as a commensal organism in the oral cavity and respiratory tract [1]. The microorganism is an opportunistic pathogen in immunocompromised patients and has been implicated in several infections, including bacteremia, meningitis, pneumonia, arthritis, endocarditis, and/or osteomyelitis $[2,3]$. Although meningitis due to $R$. mucilaginosa is a rare but potentially lethal infection in patients with neutropenia, there is a dearth of investigations on meningitis, especially in children $[3,4]$. In addition, because this infection is rare and only a few cases have been recorded, evidence-based guidelines for adequate infection treatment are lacking. Herein, we report a case of $R$. mucilaginosa meningitis in a 12 -year-old boy.

\section{Case Presentation}

A 12-year-old boy was admitted to our hospital under the diagnosis of myelodysplastic syndromes (MDS) with monosomy 7. He was treated with an allogeneic bone marrow transplant for MDS after four azacytidine cycles. As engraftment failed, he received an unrelated cord blood transplant (U-CBT). At 23 days after U-CBT, he presented with fever, vomiting, and a change in mental status. Upon physical examination, he was conscious but drowsy (Glasgow Coma Scale score of 13, E3V4M6). Neurological examinations identified a stiff neck and Kernig and Brudzinski signs. He had an aphthous ulcer on the buccal mucosa. His vital signs were as follows: body temperature, $39.7^{\circ} \mathrm{C}$; blood pressure, $112 /$ $60 \mathrm{mmHg}$; heart rate, 112 beats/min; respiratory rate, 28 breaths/min; and oxygen saturation, $100 \%$ at room air. 
Laboratory examinations revealed a decreased leukocyte count of $<100$ cells $/ \mu \mathrm{L}$. The C-reactive protein level was $13.45 \mathrm{mg} / \mathrm{dL}$, and the serum procalcitonin level was $0.79 \mathrm{ng} /$ $\mathrm{mL}$. A lumbar puncture was performed, and his cerebrospinal fluid (CSF) profile revealed 110 leukocytes/ $\mu \mathrm{L}$, with lymphocytic predominance $(67 \%)$. The CSF total protein level was $76 \mathrm{mg} / \mathrm{dL}$, and the CSF glucose level was $12 \mathrm{mg} / \mathrm{dL}$ (CSF glucose/serum glucose $=0.074)$. Microbiological Gram staining of CSF revealed only typical Gram-positive cluster forming cocci (Figure 1(a)). Two rounds of aerobic blood cultures from the peripheral vein and central venous catheter line were negative. As an empirical therapy, we commenced the treatment with meropenem (MEPM; 6g/ day) and vancomycin (VCM; $3 \mathrm{~g} /$ day). We observed that Gram-positive colonies were sticky in nature, whitish/gray, nonhemolytic, smooth, and round after $24 \mathrm{~h}$ of incubation at $37^{\circ} \mathrm{C}$ in $\mathrm{CO}_{2}$ in sheep blood agar (Figures $1(\mathrm{~b})$ and $1(\mathrm{c})$ ). Stretching of the colonies isolated from our patient's sample resulted in the formation of a string $8 \mathrm{~mm}$ in length (with a string $\geq 5 \mathrm{~mm}$ defined as positive). To corroborate this, we confirmed the organism as $R$. mucilaginosa using matrixassisted laser desorption/ionization mass spectrometry (confidence score, 2.405) at 1 day. R. mucilaginosa also grew in an oral aphtha sample.

The minimum inhibitory concentration (MIC) values of the studied antimicrobials were determined by broth microdilution. The MIC for $R$. mucilaginosa was $\leq 0.06 \mu \mathrm{g} /$ $\mathrm{mL}$ for penicillin $G, \leq 0.25 \mu \mathrm{g} / \mathrm{mL}$ for ampicillin (ABPC), $\leq 0.25 \mu \mathrm{g} / \mathrm{mL}$ for sulbactam/ampicillin, $\leq 0.25 \mu \mathrm{g} / \mathrm{mL}$ for tazobactam/piperacillin, $0.5 \mu \mathrm{g} / \mathrm{mL}$ for cefazolin (CEZ), $0.25 \mu \mathrm{g} / \mathrm{mL}$ for ceftriaxone, $1 \mu \mathrm{g} / \mathrm{mL}$ for MEPM, $1 \mu \mathrm{g} / \mathrm{mL}$ for $\mathrm{VCM}, 1 \mu \mathrm{g} / \mathrm{mL}$ for linezolid, and $4 \mu \mathrm{g} / \mathrm{mL}$ for daptomycin (DAP). We completed the VCM administration on day 3. Although the fever continued, the CSF cultures were negative on day 7. Peripheral blood neutrophil counts had recovered to $>500$ cells $/ \mu \mathrm{L}$ on day 11 . Similarly, the fever was alleviated on day 14. In total, we performed antimicrobial treatments due to MEPM therapy for 43 days. During follow-up, the patient remained afebrile and had no neurological complications, including hearing loss.

\section{Discussion}

We present the case of a 12 -year-old boy diagnosed as having R. mucilaginosa meningitis, MDS, and febrile neutropenia. Bone marrow transplant or chemotherapy in the treatment of MDS induces not only long-term neutropenia but also extensive mucositis. Chavan et al. reported 11 children who developed clinically significant $R$. mucilaginosa infections, including three deaths, directly attributable to the microorganism [5]. Almost all patients had severe neutropenia, central-line catheters, and mucosal breakdown at the time of infection. They described that $R$. mucilaginosa can lead to life-threatening infections in immunocompromised hosts, especially in profoundly neutropenic patients $[2,4,5]$. Our patient had the risk factors of $R$. mucilaginosa infection, such as severe neutropenia, central-line catheter use, and mucosal breakdown at the time of infection, that were reported in previous studies $[5,6]$.
Recently, high-dose ampicillin, third-generation cephalosporins, rifampin, chloramphenicol, and VCM have been reported to be active in the treatment of invasive R. mucilaginosa infections $[2,4,7,8]$. As $R$. mucilaginosa meningitis is rare and only a few cases have been reported, no guidelines have been established to assist in antibiotic selection for the treatment of invasive Rothia infections. Accordingly, the most effective antibiotics for R. mucilaginosa meningitis are unknown. Choosing adequate antibiotics is important because antimicrobial agents penetrate the blood-brain barrier less when little or no meningeal inflammation is present in patients with neutropenia. In addition, repeated or prolonged exposure to prophylactic or therapeutic broad-spectrum antibiotics may lead to the selection of pathogenic germs [4]. These factors make $R$. mucilaginosa meningitis treatment more difficult.

Lee et al. described the clinical characteristics of 16 immunocompromised patients who developed meningitis from $R$. mucilaginosa [4]. On the basis of a literature review, they recommended the addition of high-dose ampicillin and rifampin as first-line therapies against $R$. mucilaginosa meningitis. Ramanan et al. also reported that all $R$. mucilaginosa isolates were susceptible to penicillin, MEPM, and VCM, but 33\% were oxacillin resistant [2]. However, recently, Kayman et al. reported that isolates from patients with bacteremia were resistant to beta-lactams and susceptible to VCM [9]. Moreover, Getzenberg et al. also reported that the antibiotic susceptibility of $R$. mucilaginosa isolates were $3 \%$ for penicillin, $0 \%$ for oxacillin, $76 \%$ for CEZ, 73\% for MEPM, and 100\% for VCM [7]. All the cases were successfully treated with VCM. Maraki et al. reviewed 19 published cases $(n=20)$ of $R$. mucilaginosa pneumonia. VCM alone or in combination with other antibiotics have been successfully used for the treatment of $R$. mucilaginosa pneumonia [10]. On the basis of these data, they recommended the administration of VCM as a first-line therapy against invasive $R$. mucilaginosa infection cases.

From now on, owing to the increasing number of reported cases of relative penicillin resistance, other agents are increasingly needed. DAP is used to treat patients with GPC bacteremia or meningitis as empirical therapy. Recently, because $R$. mucilaginosa isolates with high MICs to DAP were observed, caution is required when treating R. mucilaginosa infection with DAP. Bruminhent et al. described that the MIC of DAP was $2.0 \mu \mathrm{g} / \mathrm{mL}$ in their case isolate [11]. We retrospectively reviewed the antimicrobial susceptibility of all $R$. mucilaginosa isolates $(n=5)$ from blood or CSF cultures at our hospital between January 2016 and December 2020 (Table 1), revealing that some strains were resistant to $\beta$-lactam antibiotics, including $\mathrm{ABPC}$. Moreover, the MICs of VCM and LZD were $<0.5 \mu \mathrm{g} / \mathrm{mL}$ for all strains, whereas the MIC of DAP was $\geq 2 \mu \mathrm{g} / \mathrm{mL}$ for all strains. The MIC of MEPM was $>1 \mu \mathrm{g} / \mathrm{mL}$ for one strain.

In our case series, the antibiotic susceptibility of R. mucilaginosa isolates was $60 \%$ for penicillin, $60 \%$ for ABPC, $60 \%$ for CEZ, $80 \%$ for MEPM, and 100\% for VCM. The antimicrobial susceptibility results differed among the isolates. Breakthrough GPC bacteremia or meningitis in patients treated with $\beta$-lactam antibiotics or DAP may reflect 




(a)



(b)

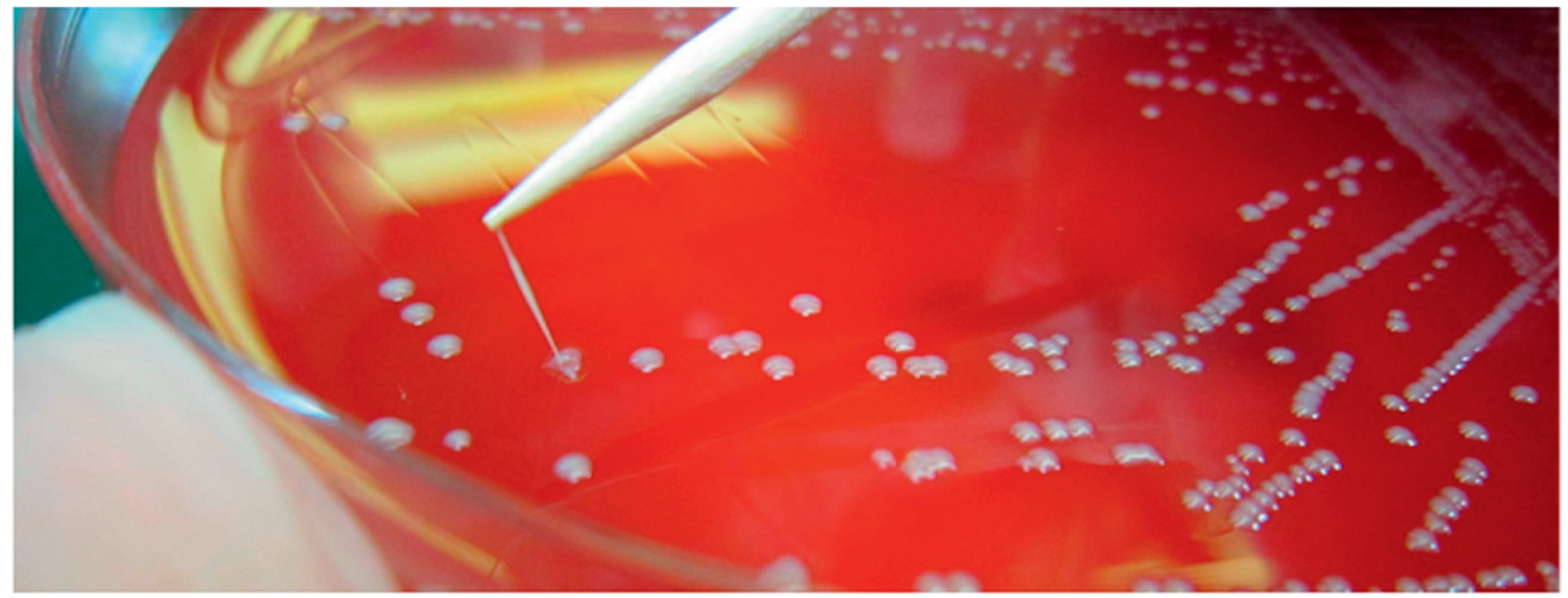

(c)

FIgURE 1: Macroscopic and microscopic appearance of Rothia mucilaginosa. (a) Gram staining of characteristic blue cocci from a cerebrospinal fluid culture at $37^{\circ} \mathrm{C}$ for $24 \mathrm{~h}$. (b) A blood agar plate showing R. mucilaginosa colonies in a cerebrospinal fluid sample from the patient, cultured over $24 \mathrm{~h}$ at $37^{\circ} \mathrm{C}$ in $\mathrm{CO}_{2}$. (c) Sticky and slimy colonies and a positive string test result.

TABLE 1: Clinical features and distribution of the MICs against antimicrobials of Rothia mucilaginosa isolated in our hospital.

\begin{tabular}{|c|c|c|c|c|c|}
\hline & Case 1 & Case 2 & Case 3 & Case 4 & Case $5^{*}$ \\
\hline Age/sex & $81 \mathrm{y} / \mathrm{F}$ & $38 \mathrm{y} / \mathrm{F}$ & $7 \mathrm{y} / \mathrm{M}$ & $4 \mathrm{y} / \mathrm{M}$ & $12 \mathrm{y} / \mathrm{M}$ \\
\hline Underlying disease & Lymphoma & AML & Lymphoma & ALL & MDS \\
\hline Culture source & Blood & Blood & Blood & Blood & CSF \\
\hline Infection & Sepsis & Sepsis & Sepsis & Sepsis & Meningitis \\
\hline \multicolumn{6}{|l|}{$\operatorname{MIC}(\mu \mathrm{g} / \mathrm{mL})$} \\
\hline Penicillin G & $\leqq 0.06$ & $>2$ & $>2$ & $\leqq 0.06$ & $\leqq 0.06$ \\
\hline Ampicillin & $\leqq 0.25$ & $>4$ & $>4$ & $\leqq 0.25$ & $\leqq 0.25$ \\
\hline Cefazolin & 0.25 & $>2$ & $>2$ & 0.5 & 0.5 \\
\hline Ceftriaxone & $\leqq 0.12$ & $>2$ & $>2$ & 0.25 & 0.5 \\
\hline Meropenem & $\leqq 0.06$ & $>1$ & 1 & 0.5 & 1 \\
\hline Levofloxacin & $>4$ & $>4$ & 1 & $>4$ & 1 \\
\hline
\end{tabular}


TABLE 1: Continued.

\begin{tabular}{lccccc}
\hline & Case 1 & Case 2 & Case 3 & Case 4 & Case $^{*}$ \\
\hline Vancomycin & $\leqq 1$ & 1 & 1 & 1 & 1 \\
Teicoplanin & $\leqq 1$ & $\leqq 0.5$ & $\leqq 0.5$ & 2 & $\leqq 0.5$ \\
Daptomycin & 4 & 4 & 0.5 & 2 & $\leq 0.5$ \\
Linezolid & 1 & 1 & 1 & 4 \\
\hline
\end{tabular}

*Presented case. ALL, acute lymphoid leukemia; AML, acute myeloid leukemia; CSF, cerebrospinal fluid; F, female; M, male; MDS, myelodysplastic syndromes; MIC, minimum inhibitory concentration; $y$, year.

emergence of $\beta$-lactam antibiotics or DAP nonsusceptibility in the infecting isolate.

Different results regarding the antibiotic susceptibility of $R$. mucilaginosa have been reported by various authors [2-11]. However, the reasons for the differences in the antibiotic susceptibility pattern among $R$. mucilaginosa isolates are not clear. Further research is needed to analyze microbiological and clinical features, including underlying disease, antimicrobial treatment, or oral and gastrointestinal flora.

In summary, we recommend that VCM should be chosen as the primary treatment for invasive $R$. mucilaginosa infections until antimicrobial susceptibility results are known, especially in immunocompromised children.

\section{Consent}

Informed consent for publishing this case report was obtained from the patient's parents.

\section{Conflicts of Interest}

The authors declare that they have no conflicts of interest.

\section{Authors' Contributions}

FO, RN, RM, and KM managed the patient and prepared the manuscript. SM performed the biochemical and mass spectrometry analyses. HT reviewed the manuscript. All authors read and approved the final manuscript.

\section{References}

[1] M. Z. Abidi, N. Ledeboer, A. Banerjee, and P. Hari, "Morbidity and mortality attributable to Rothia bacteremia in neutropenic and nonneutropenic patients," Diagnostic Microbiology and Infectious Disease, vol. 85, no. 1, pp. 116-120, 2016.

[2] P. Ramanan, J. N. Barreto, D. R. Osmon, and P. K. Tosh, "Rothia bacteremia: a 10 year experience at mayo clinic, rochester, Minnesota," Journal of Clinical Microbiology, vol. 52, no. 9, pp. 3184-3189, 2014.

[3] M. Clauwaert, P. Druwé, and P. Depuydt, "Meningitis in a patient with neutropenia due to Rothia mucilaginosa: a case report," Journal of Medical Case Reports, vol. 13, no. 1, p. 84, 2019.

[4] A. B. Lee, P. Harker-Murray, P. Ferrieri, M. R. Schleiss, and J. Tolar, "Bacterial meningitis from Rothia mucilaginosa in patients with malignancy or undergoing hematopoietic stem cell transplantation," Pediatric Blood and Cancer, vol. 50, no. 3, pp. 673-676, 2008.
[5] R. S. Chavan, P. S. Pannaraj, R. A. Luna et al., "Significant morbidity and mortality attributable toRothia MucilaginosaInfections in children with hematological malignancies or following hematopoietic stem cell transplantation," Pediatric Hematology and Oncology, vol. 30, no. 5, pp. 445-454, 2013.

[6] A. J. Kaasch, G. Saxler, and H. Seifert, "Septic arthritis due to Rothia mucilaginosa," Infection, vol. 39, no. 1, pp. 81-82, 2011.

[7] R. B. Getzenberg, D. R. Hijano, H. Hakim et al., "Rothia mucilaginosa infections in pediatric cancer patients," Journal of the Pediatric Infectious Diseases Society, vol. 10, 2020.

[8] C. Von Eiff, M. Herrmann, and G. Peters, "Antimicrobial susceptibilities of Stomatococcus mucilaginosus and of Micrococcus spp," Antimicrobial Agents and Chemotherapy, vol. 39, no. 1, pp. 268-270, 1995.

[9] T. Kayman, T. Akalin, H. Ugur, B. Bozdogan, and S. Duyan, "Two bacteremia cases associated with Rothia mucilaginosa," Clinical Laboratory, vol. 59, pp. 1167-1170, 2013.

[10] S. Maraki and I. S. Papadakis, "Rothia mucilaginosa pneumonia: a literature review," Infectious Diseases, vol. 47, no. 3, pp. 125-129, 2015.

[11] J. Bruminhent, M. J. Tokarczyk, D. Jungkind, and J. A. DeSimone, "Rothia mucilaginosa prosthetic device infections: a case of prosthetic valve endocarditis," Journal of Clinical Microbiology, vol. 51, no. 5, pp. 1629-1632, 2013. 